\title{
Assembly and Disassembly Mechanics of a Cylindrical Snap Fit
}

\author{
Xiao-Lin Guo $^{1}$ and Bo-Hua Sun ${ }^{* 2}$ \\ ${ }^{1}$ School of Science 85 Institute of Mechanics and Technology \\ ${ }^{2}$ School of Civil Engineering 83 Institute of Mechanics and Technology \\ Xi'an University of Architecture and Technology, Xi'an 710055, China
}

\begin{abstract}
Snap fit is a common mechanical mechanism. It uses the physical asymmetry that is easy to assemble but difficult to disassemble to provide a simple and fast link between objects. The ingenious combination of geometric shape, bending elasticity and friction of the snap fit is the mechanism behind the easy to assemble but difficult to disassemble disassemble. Yoshida and Wada (2020) has done a groundbreaking work in the analysis of the elastic snap fit. During our study of their paper, while we really enjoyed their research, unfortunately we detected several questioning formulations. After careful checking, we found that those formulations are not typographical, therefore it is necessary to make corrections. This paper reformulates the linear elasticity of a cylindrical snap fit, obtains an exact solution and proposes an accurate relation between the opening angle and bending tangent angle. Under the first order approximation, our formulations can reduced to the results of Yoshida and Wada and hence confirms the scientific correctness of Yoshida and Wada's work. Furthermore, this paper also derives a correct vertical displacement expression, and propose a new way of disassembly by bending for the first time and formulate a scaling law by data fitting. All formulations are validated by finite element simulation and experiment. The research here is helpful to the design of elastic snap fit or adjustable mechanical mechanism and metamaterial cell.
\end{abstract}

Keywords: Snap fit, elasticity, friction, geometry, beam, symmetry breaks

\section{Introduction}

The snap fit can be seen everywhere in our life because it is simple, convenient and reusable (Fig. 1). The two objects can be directly connected without welding, bolts, glue and other means, and can be assembled and disassembled repeatedly [1]. There are vast varieties of snap fits ranging from receptor ligand interaction in biochemistry [2] to spacecraft docking in the space [3], as well as zippers, Lego blocks and water pipe clamps commonly used in life [1-20] . Although the buckle is not uncommon for snap fit, it implies a basic physical mechanism, that is, the buckle makes use of the mechanical asymmetry that is easy to assemble but difficult to disassemble.
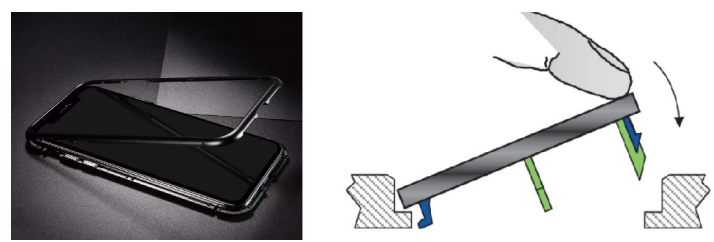

FIG. 1: Elastic cantilever snap fit

Although we often hear the "click" sound of the snap fit in our daily life, there is very little research on the scientific mechanism of the snap fit mechanism. In 2020, Yoshida and Wada [4] creatively conducted a compressive study and gave some basic relationships and phase diagram on the assembly and disassembly mechanics of

\footnotetext{
*Corresponding author: Bo-Hua Sun, email: sunbohua@xauat.edu.cn
}

the cylindrical snap fit as shown in Fig. 2. Their research has open a new door to accurately predict the mechanism of snap fit from physics point of view. The definition of all notations can be found in the next section.
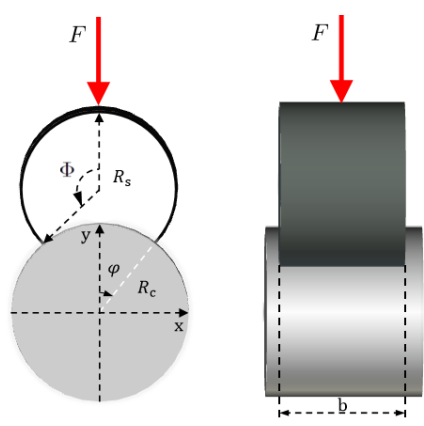

FIG. 2: Cylindrical snap fit

The upmost relationship of the snap fit device is the formulation between the snap fit bending rotation angle $\theta_{0}$ and the snap fit initial opening angle $\Phi$, i.e

$$
\theta_{0}=\theta_{0}(\Phi)
$$

Yoshida and Wada [4] did not give an exact solution, but an approximate expression without any interpretation:

$$
\theta_{0}=\Phi-(\sin \Phi-\Phi \cos \Phi) \epsilon+O\left(\epsilon^{2}\right)
$$

This expression is the Eq.(S6) in Yoshida and Wada's supplement [5]. Then they derived all other formulas in the paper by using the expression (2). In the supplementary material [5], Yoshida and Wada did not show how to get the expression (2), nor the error analysis verification of expression (2). 
According to rigorous mathematics, the first-order approximation relationship between snap fit bending cutting angle and initial opening angle should be as follows:

$$
\Phi=\theta_{0}+\left(\sin \theta_{0}-\theta_{0} \cos \theta_{0}\right) \epsilon+O\left(\epsilon^{2}\right)
$$

We can immediately recognise that this expression is different from the expression (2), but why?

Since all other expressions of snap fit mechanics require the use of expressions (2), whose correctness is crucial, therefore, it is reasonable to ask whether the results of Yoshida and Wada [4] can be trusted. In order to answer this question, it is necessary to reformulate all expressions in the paper of Yoshida and Wada [4]. If the reformulation study finds problems in the results of Yoshida and Wada [4], it should be corrected. If the results of Yoshida and Wada [4] are found to be reasonable, it would be a scientific affirmation of the work of Yoshida and Wada [4].

In this paper, we first introduced the assembly and disassembly mechanism of the cylindrical snap fit, and then deduced its theoretical model, obtains the exact solution of the relationship and its first order approximation between the snap fit bending angle and the initial opening angle, based on which we formulate the assembly force and disassembly force; finite element simulation is carried out on the two types of snap fit for pushing assembly and pulling disassembly, as well as for pushing assembly and bending disassembly. In order to have a deeper understanding of physics, experimental analysis and comparisons are made. Finally, a discussion is made and a conclusion is drawn.

\section{ASSEMBLY AND DISASSEMBLY MECHANISM OF SNAP FIT}

As shown in Figs.2 and 3, we adopt a semi-cylindrical shell with radius of $R_{s}$, thickness of $t$, length of $b$ and opening angle of $\Phi$. It is pushed onto the surface of a rigid cylinder with radius of $R_{c}$ to form a simple cylindrical snap fit.

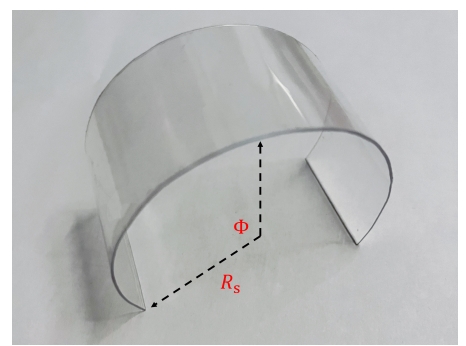

FIG. 3: Snap fit in natural condition

When the natural state snap fit is pushed down, the snap fit slides along the surface of the rigid cylinder under the combined action of thrust and friction. In the initial stage, the thrust is gradually increased to push the buckle to slide. When a critical point is reached, the snap fit moves to the final configuration driven by its own elastic bending force without any more additional pushing.

According to the interactions between the topology, bending elasticity and friction that are characterised by the initial opening angle $\Phi$, mismatch ratio $\alpha=R_{c} / R_{s}$, elasticity modulus $E$ and friction coefficient $\mu$, the assembly process of the snap fit can be vary, corresponding to different physical phenomena. One is sliding assembly and the other is jumping assembly. Correspondingly the snap fit can be divided into two types: Type I and Type II snap fit.

Type I snap fit has smaller initial opening angle $\Phi$, and its assembly and disassembly are relative easier. The assembly and disassembly of Type I snap fit are generally in symmetric deformation as shown in Fig.4.

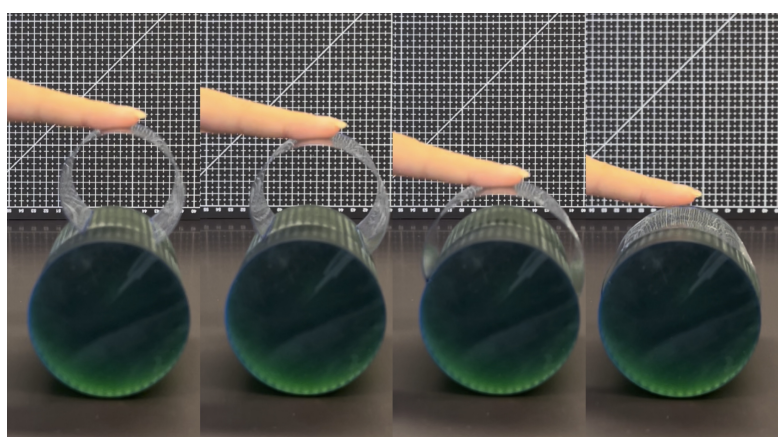

FIG. 4: Type I snap fit

Type II snap fit has larger initial opening angle $\Phi$, so it is difficult to assemble and disassemble. In the assembly process of Type II snap fit, the snap fit is generally in asymmetric deformation, especially in the installation process, the snap fit begins to have large symmetric deformation, and then suddenly has asymmetric deformation to realize assembly, as shown in Fig.5. Conversely, in the disassembly process of Type II snap fit, the snap fit can generally be considered to be in symmetrical deformation.

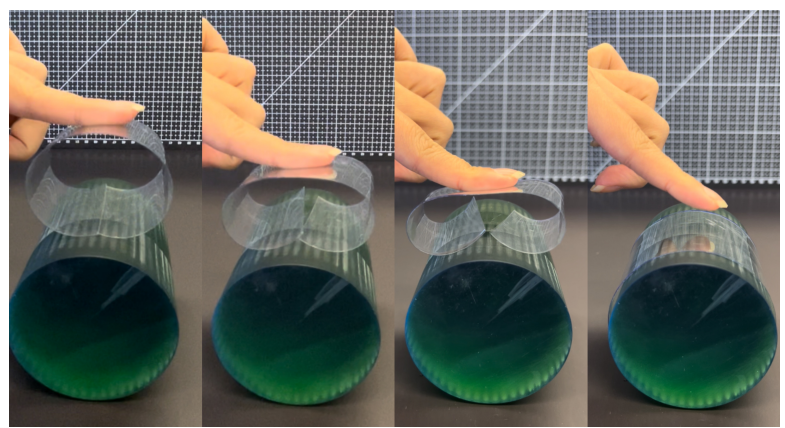

FIG. 5: Type II snap fit

From the above description, the mechanics nature of a fully assembled snap fit is easy to assembly but difficult to disassembly (i.e. within the initial opening angle), that 
is $\left|F_{D}\right| / F_{A}>1$, where $F_{A}$ represents the assembly force of the snap fit and $F_{D}$ represents the disassembly force of the snap fit, which is a very interesting asymmetric physical phenomenon. It is anticipated to have energy dissipation of the assembly-disassembly circle. Therefore, the snap fit is a good platform for symmetric breaks and energy dissipation (or harvesting) testing.

Snap fit assembly has a critical point, before this point if the thrust is removed, the snap fit will rebound back under its own bending elastic drive, can not complete the snap fit installation. Beyond this point, if the thrust is removed, the snap fit will continue to complete the snap fit installation under its own flexural elastic drive.

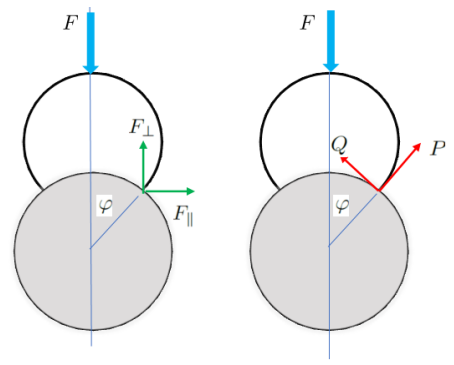

FIG. 6: Loading of snap fit

Driven by the force of $F$, the cylinder surface has reaction force on the buckle: normal force $P$ and tangential force $Q$. According to the Fig.6, the balance equation in the direction of $y$ is: $2 P \cos \varphi+2 Q \sin \varphi=F$. According to Amontons Coulomb's friction theorem, the relation $Q=\mu P$. Simultaneous solution can be obtained

$$
P=\frac{F}{2} \frac{1}{\cos \varphi+\mu \sin \varphi}, \quad Q=\frac{\mu F}{2} \frac{1}{\cos \varphi+\mu \sin \varphi},
$$

According to the force balance condition of $y$, we can get $F_{\perp}=\frac{F}{2}$. Then according to the force balance condition of $x$, can be obtained

$$
F_{\|}=P \sin \varphi-Q \cos \varphi=\frac{F}{2} \frac{\tan \varphi-\mu}{1+\mu \tan \varphi}
$$

A similar formula is valid for disassembling process, with the replacement given by $\mu \rightarrow-\mu$ in Eq.5.

The critical point can be obtained from the condition $1+\mu \tan \varphi^{*}=0$, which leads to $\varphi^{*}=\arctan (-1 / \mu)$, or critical opening angle $\Phi^{*} \approx \alpha[\pi-\arctan (-1 / \mu)]$, which is proposed by [4].

\section{THEORY OF SNAP FIT ASSEMBLE AND DISASSEMBLE MECHANICS}

From the above discussion, we understood that the upmost basic relationship in the study of snap fit problem is how to use the initial opening angle $\Phi$ to represent the deformation rotation angle $\theta_{0}$, namely $\theta_{0}=\theta_{0}(\Phi)$.
Yoshida and Wada [4] directly gave the first order analytical expression $\theta_{0}=\theta_{0}(\Phi)$ as follows

$$
\theta_{0}=\Phi-(\sin \Phi-\Phi \cos \Phi) \epsilon+O\left(\epsilon^{2}\right)
$$

As we have pointed out in the previous section, since Yoshida and Wada did not deduce the expression (6) in the supplementary material [5] and did not give the error analysis and verification of the expression (6), we have a good reason to ask whether the results of Yoshida and Wada [4] is credible? To answer this question, let's deduce the relevant expression again.

Denoting the snap fit has natural state as radius $R_{s}$, thickness $t$, length $b$ and opening angle $\Phi$, the snap fit is pushed to radius $R_{c}$ on the surface of a rigid cylinder, will create a simple cylindrical snap fit, as shown in Fig.2 and Fig.7.

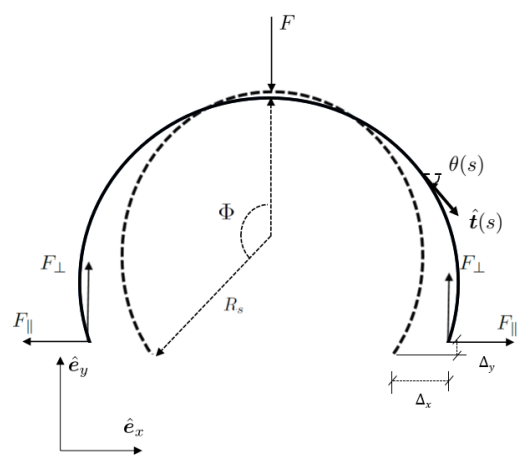

FIG. 7: Schematic diagram of cylindrical snap fit

The snap fit is assembled on a rigid cylinder, and there is no deformation in the length direction of the cylinder, namely length inextensible. We only need to study the deformation of the shape of its cross section, so that the problem is transformed from three-dimensional to twodimensional, and the cylindrical shell problem is simplified to the problem of elastic circular rod or beam. Considering the small deformation of a naturally curved elastomer with a constant arc length $L=2 R_{s} \Phi$, which is affected by the horizontal force $F_{\|}$and the longitudinal force $F_{\perp}$ applied at the edge The resultant force vector is $\boldsymbol{F}=\boldsymbol{F}_{\|} \hat{\boldsymbol{e}}_{x}+\boldsymbol{F}_{\perp} \hat{\boldsymbol{e}}_{y}$.

We assume that 2D symmetric deformation occurs in the $x-y$ plane, and its horizontal displacement $\Delta_{x}$ is shown in Fig.7. Through its arc length parameter $s$ (starting from the top), we use the unit tangent vector $\hat{\boldsymbol{t}}(s)=\cos \theta(s) \hat{\boldsymbol{e}}_{x}-\sin \theta(s) \hat{\boldsymbol{e}}_{y}$, as shown in Fig.7. In the absence of any external forces and moments, the balance equation is given by [20]:

$$
\frac{d \boldsymbol{F}(s)}{d s}=0, \quad \frac{\boldsymbol{M}(s)}{d s}+\hat{\boldsymbol{t}}(s) \times \boldsymbol{F}(s)=0,
$$

Where $\boldsymbol{F}(s)$ and $\boldsymbol{M}(s)$ are internal forces and elastic moments.

We assume a linear constitutive relation (i.e. Hooke's law) $M(s)=B\left(\kappa(s)-\kappa_{0}\right) \hat{\boldsymbol{e}}_{z}$, where $B=E I$ is the 
bending modulus, $E$ is the elastic modulus, and $I$ is the moment of inertia of the snap fit cross-section. $\kappa(s)=$ $-\theta^{\prime}(s)$ is the curvature, $\kappa_{0}=-1 / R_{s}(<0)$ is the natural curvature of elasticity.

By integrating the first equation of equation 7 and using the boundary condition: $F(0)=0$, it can be obtained $F=\boldsymbol{F}_{\|} \hat{\boldsymbol{e}}_{x}+\boldsymbol{F}_{\perp} \hat{\boldsymbol{e}}_{y}$. Then it is brought into the second equation of equation ( refeq-1) and pay attention to the operation $\hat{\boldsymbol{t}}(s) \times$ $\boldsymbol{F}(s)=\left[\cos \theta(s) \hat{\boldsymbol{e}}_{x}-\sin \theta(s) \hat{\boldsymbol{e}}_{y}\right] \times\left(F_{\|} \hat{\boldsymbol{e}}_{x}+\boldsymbol{F}_{\perp} \hat{\boldsymbol{e}}_{y}\right)=$ $\cos \theta(s) F_{\|} \underbrace{\hat{\boldsymbol{e}}_{x} \times \hat{\boldsymbol{e}}_{x}}_{=0}-\sin \theta(s) F_{\|} \underbrace{\hat{\boldsymbol{e}}_{y} \times \hat{\boldsymbol{e}}_{x}}_{=-\hat{\boldsymbol{e}}_{z}}+F_{\perp} \cos \theta(s) \hat{\boldsymbol{e}}_{z}=$ $\left(F_{\|} \sin \theta(s)+F_{\perp} \cos \theta(s)\right) \hat{\boldsymbol{e}}_{z}$, we have $\frac{d}{d s}\left[B\left(\kappa-\kappa_{0}\right)\right] \hat{\boldsymbol{e}}_{z}=$ $\left(F_{\|} \sin \theta(s)+F_{\perp} \cos \theta(s)\right) \hat{\boldsymbol{e}}_{z}$, namely, the elastic equation of snap fit is

$$
B \frac{d^{2} \theta(s)}{d s^{2}}=F_{\|} \sin \theta(s)+F_{\perp} \cos \theta(s) .
$$

boundary conditions: $\theta(L / 2) \equiv \theta_{0}$ and $\theta^{\prime}(L / 2)=-\kappa_{0}=$ $1 / R_{s}$, where $L=2 R_{s} \Phi$.

\section{A. Snap fit elasticity under horizontal force}

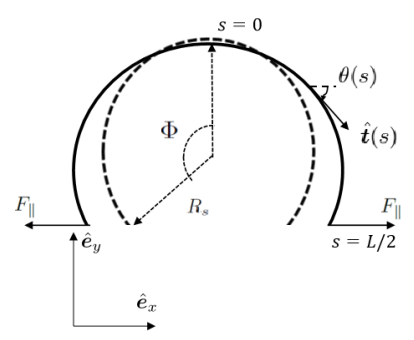

FIG. 8: Snap fit under horizontal force $F_{\|}$

In Fig.8, if only horizontal force $F_{\|}$, longitudinal force $F_{\perp}=0$, Eq. (8) degenerates into

$$
B \frac{d^{2} \theta(s)}{d s^{2}}=F_{\|} \sin \theta(s),
$$

together with the relevant boundary conditions : $\theta(L / 2) \equiv \theta_{0}$ and $\theta^{\prime}(L / 2)=-\kappa_{0}=1 / R_{s}$, where $L=2 R_{s} \Phi$.

Applying boundary conditions, the first integral elastic equation is calculated as follows:

$$
\left(\frac{d \theta(s)}{d s}\right)^{2}=\frac{1}{R_{s}^{2}}+2 \frac{F_{\|}}{B}\left(\cos \theta_{0}-\cos \theta\right)
$$

it can be rewritten as

$$
\frac{d s}{R_{s}}=\frac{d \theta}{\sqrt{1-2 \epsilon\left(\cos \theta-\cos \theta_{0}\right)}}
$$

where the small parameter $\epsilon$ is $\epsilon=\frac{F_{\|} R_{s}^{2}}{B}$.
Substitute equation (11) into the non elongation condition $\int_{0}^{L / 2} d s=R_{s} \Phi$ of the elastomer, where $L=2 R_{s} \Phi$. The non elongation condition can be written as

$$
\int_{0}^{L / 2} d s=\int_{0}^{\theta_{0}} \frac{d \theta}{\sqrt{1-2 \epsilon\left(\cos \theta-\cos \theta_{0}\right)}} .
$$

For any parameter $\epsilon=\frac{F_{\|} R_{s}^{2}}{B}$, by a symbolic computation such as Maple, we can find the exact solution of equation (12) as follows

$$
\Phi=2 \frac{\text { InverseJacobsAM }\left(\frac{\theta_{0}}{2}, 2 \sqrt{\frac{\epsilon}{2 \epsilon\left(1-\cos \theta_{0}\right)-1}}\right)}{\sqrt{1+2 \epsilon\left(\cos \theta_{0}-1\right)}},
$$

where InverseJacobsAM represents the triangular form of the first kind of incomplete elliptic integral[6] and clearly the condition $\cos \theta_{0} \geq 1-\frac{1}{2} \frac{B}{F R_{s}^{2}}$ must be applied.

Because we only know the initial opening angle $\Phi$ and do not know the inclination angle $\theta_{0}$ of deformation. In order to invert the above exact solution and obtain the analytical expression of deformation inclination $\Phi$ expressed by initial opening angle $\theta_{0}$, i.e. $\theta_{0}=\theta_{0}(\Phi)$.

Although we obtained the exact solution in Eq.13, to the best of the authors' knowledge, it is almost impossible have the inversion of the exact solution. We have to give up the exact solution. For convenient purpose, let's try whether the approximate treatment is feasible.

Expanding the expression (11) for the small parameter $\epsilon=\frac{F_{\|} R_{s}^{2}}{B}$ and take the first-order approximation

$$
\frac{d s}{R_{s}} \approx d \theta\left[1+\epsilon\left(\cos \theta-\cos \theta_{0}\right)+O\left(\epsilon^{2}\right) .\right.
$$

Substituting the expression (14) into the non elongation condition $\int_{0}^{L / 2} d s=R_{s} \Phi$ of the elastomer, where $L=$ $2 R_{s} \Phi$. the non elongation condition can be written as

$$
\int_{0}^{L / 2} d s=\int_{0}^{\theta_{0}}\left[d \theta\left[1+\epsilon\left(\cos \theta-\cos \theta_{0}\right)+O\left(\epsilon^{2}\right)\right]\right.
$$

can find

$$
\Phi=\theta_{0}+\left(\sin \theta_{0}-\theta_{0} \cos \theta_{0}\right) \epsilon+O\left(\epsilon^{2}\right) .
$$

It is clear that this expression is different from expression (6). Here expression (16) is a first-order approximate result derived naturally by rigorous mathematics. According to this expression, we can deduce other following results of assembly and disassembly.

\section{Horizontal displacement caused by $F_{\|}$}

It can be seen from the deformation in Fig. 7 that the horizontal deformation relationship is

$$
x(L / 2)-x(0)=\int_{0}^{L / 2} \cos \theta(s) d s=R_{s} \sin \Phi+\Delta_{x \|},
$$


where $\Delta_{x \|}$ represents the horizontal displacement driven by force $F_{\|}$.Substitute expression (14) into (17) to get

$$
\begin{aligned}
& x(L / 2)-x(0) \\
& =R_{s} \int_{0}^{\theta_{0}} \cos \theta(s)\left[1+\epsilon\left(\cos \theta-\cos \theta_{0}\right)\right] d \theta+O\left(\epsilon^{2}\right) \\
& \approx R_{s} \sin \Phi+\Delta_{x \|} x .
\end{aligned}
$$

or write

$$
\sin \theta_{0}+\frac{1}{2} \epsilon\left(\theta_{0}-\sin \theta_{0} \cos \theta_{0}\right)=\sin \Phi+\frac{\Delta_{x \|}}{R_{s}} .
$$

Substituting the expression (16) into the above formula to obtain

$$
\begin{aligned}
& \sin \theta_{0}+\frac{1}{2} \epsilon\left(\theta_{0}-\sin \theta_{0} \cos \theta_{0}\right) \\
& =\sin \left[\theta_{0}+\left(\sin \theta_{0}-\theta_{0} \cos \theta_{0}\right) \epsilon\right]+\frac{\Delta_{x \|}}{R_{s}},
\end{aligned}
$$

Expanding the both sides to the first-order term of $\epsilon$ leads to

$$
\begin{aligned}
& \sin \theta_{0}+\frac{1}{2} \epsilon\left(\theta_{0}-\sin \theta_{0} \cos \theta_{0}\right) \\
& =\frac{\Delta_{x}}{R_{s}}+\sin \theta_{0}-\cos \theta_{0}\left(\cos \theta_{0} \theta_{0}-\sin \theta_{0}\right) \epsilon,
\end{aligned}
$$

we can get $\epsilon=F_{\|} R_{s}^{2} / B$ expression

$$
\frac{F_{\|} R_{s}^{2}}{B}=K_{x \|}\left(\theta_{0}\right) \frac{\Delta_{x \|}}{R_{s}},
$$

where the elastic coefficient is defined as

$$
\begin{array}{r}
K_{x \|}\left(\theta_{0}\right)=\left[\frac{\theta_{0}}{2}-\left(\frac{3}{2} \sin \theta_{0}-\theta_{0} \cos \theta_{0}\right) \cos \theta_{0}\right]^{-1} . \\
\text { 2. } \text { Vertical displacement generated by } F_{\|}
\end{array}
$$

It can be seen from deformation in Fig.7 that the longitudinal deformation relationship is

$y(L / 2)-y(0)=\int_{0}^{L / 2} \sin \theta(s) d s=R_{s}(1-\cos \Phi)+\Delta_{y \|}$,

where $\Delta_{y \|}$ represents the vertical displacement contributed by $F_{\|}$.

The expression (14) is substituted into (24) to obtain

$$
\begin{aligned}
& x(L / 2)-x(0) \\
& =R_{s} \int_{0}^{\theta_{0}} \cos \theta(s)\left[1+\epsilon\left(\cos \theta-\cos \theta_{0}\right)\right] d \theta+O\left(\epsilon^{2}\right) \\
& \approx R_{s}(1-\cos \Phi)+\Delta_{y \|} .
\end{aligned}
$$

it can also be written as

$$
\begin{aligned}
& 1-\cos \theta_{0}+\frac{1}{2} \epsilon\left[\frac{1}{2} \cos ^{2} \theta_{0}-\frac{1}{2}+\left(\cos \theta_{0}-1\right) \cos \theta_{0}\right] \\
& =1-\cos \Phi+\frac{\Delta_{y \|}}{R_{s}} .
\end{aligned}
$$

Substituting the expression (16) into the above equation to obtain

$$
\begin{aligned}
& -\cos \theta_{0}+\frac{1}{2} \epsilon\left(\frac{3}{2} \cos ^{2} \theta_{0}-\cos \theta_{0}-\frac{1}{2}\right) \\
& =-\cos \left[\theta_{0}+\left(\sin \theta_{0}-\theta_{0} \cos \theta_{0}\right) \epsilon\right]+\frac{\Delta_{y \|}}{R_{s}} .
\end{aligned}
$$

Expanding the both sides to the first-order term of $\epsilon$ leads to

$$
\begin{aligned}
& -\cos \theta_{0}+\frac{1}{2} \epsilon\left(\frac{3}{2} \cos ^{2} \theta_{0}-\cos \theta_{0}-\frac{1}{2}\right) \\
& =-\cos \theta_{0}+\left(\sin \theta_{0}-\theta_{0} \cos \theta_{0}\right) \epsilon+\frac{\Delta_{y \|}}{R_{s}} .
\end{aligned}
$$

we can get $\epsilon=F_{\|} R_{s}^{2} / B$ expression

$$
\frac{F_{\|} R_{s}^{2}}{B}=K_{y \|}\left(\theta_{0}\right) \frac{\Delta_{y \|}}{R_{s}},
$$

where the elastic coefficient is defined a

$$
K_{y \|}\left(\theta_{0}\right)=\left[1-\cos \theta_{0}-\frac{5}{2} \sin ^{2} \theta_{0}+\theta_{0} \sin \theta_{0} \cos \theta_{0}\right]^{-1} \text {. }
$$

\section{B. Snap fit elasticity under vertical load $F_{\perp}$}

If there is no horizontal force $F_{\|}$but only a longitudinal force $F_{\perp}$, the equation (8) above degenerates into $B \theta^{\prime \prime}(s)-F_{\perp} \cos \theta(s)=0$ and the integral can be obtained: $\frac{1}{2} B\left(\theta^{\prime}\right)^{2}-F_{\perp} \sin \theta=C$, using boundary conditions $\theta(L / 2) \equiv \theta_{0}$ and $\theta^{\prime}(L / 2)=-\kappa_{0}=1 / R_{s}$, where $L=$ $2 R_{s} \Phi$, we get the integral constant $C=\frac{1}{2} \frac{B}{R_{s}^{2}}-F_{\perp} \sin \theta_{0}$. Hence we have

$$
\frac{d s}{R_{s}}=\frac{d \theta}{\sqrt{1+2 \varepsilon\left(\sin \theta-\sin \theta_{0}\right)}}
$$

where $\varepsilon=\frac{F_{\perp} R_{s}^{2}}{B}$. We can also get the above integration exact solution, however, it is not user friendly, we will adopt approximation approach as previous section. For small $\varepsilon=\frac{F_{\perp} R_{s}^{2}}{B}$, we obtain

$$
\Phi \approx \theta_{0}+\varepsilon\left(\theta_{0} \sin \theta_{0}+\cos \theta_{0}-1\right) .
$$

\section{Horizontal deformation generated by $F_{\perp}$}

Let us calculate the horizontal displacement $\Delta_{x \perp}$ contributed by $F_{\perp}$, namely

$$
x(L / 2)-x(0)=\int_{0}^{L / 2} \cos \theta d s=R_{s} \sin \Phi+\Delta_{x \perp},
$$

Approximating the expression to the 1st order of $\varepsilon$, we have

$$
\sin \theta_{0}+\varepsilon \sin ^{2} \theta_{0}=R_{s} \sin \Phi+\frac{\Delta_{x \perp}}{R_{s}}
$$


Substituting expression (32) into (34) and only keeping the 1st order of $\varepsilon$, and solving $\varepsilon$ gives

$$
\frac{F_{\perp} R_{s}^{2}}{B}=K_{x \perp}\left(\theta_{0}\right) \frac{\Delta_{x \perp}}{R_{s}},
$$

where the stiffness

$$
K_{x \perp}\left(\theta_{0}\right)=\left[\frac{1}{2}+\cos \theta_{0}-\frac{3}{2} \cos ^{2} \theta_{0}-\theta_{0} \sin \theta_{0} \cos \theta_{0}\right]^{-1} \text {. }
$$

\section{Vertical deformation generated by $F_{\perp}$}

Let us calculate the horizontal deformation $\Delta_{y \perp}$ contributed by $F_{\perp}$, namely

$$
y(L / 2)-y(0)=\int_{0}^{L / 2} \sin \theta d s=R_{s}(1-\cos \Phi)+\Delta_{y \perp},
$$

Approximating the above expression to the 1st order of $\varepsilon$, we have

$-\cos \theta_{0}+\varepsilon\left(\sin \theta_{0}-\frac{1}{2} \sin \theta_{0} \cos \theta_{0}-\frac{1}{2} \theta_{0}\right)=-\cos \Phi+\frac{\Delta_{y \perp}}{R_{s}}$,

Substituting expression (32) into (38) and only keeping the 1 st order of $\varepsilon$, and solving $\varepsilon$ gives

$$
\frac{F_{\perp} R_{s}^{2}}{B}=K_{y \perp} \frac{\Delta_{y \perp}}{R_{s}},
$$

where the stiffness

$$
K_{y \perp}\left(\theta_{0}\right)=\left[-\frac{1}{2} \theta_{0}+\left(\frac{3}{2} \cos \theta_{0}-\theta_{0} \sin \theta_{0}\right) \sin \theta_{0}\right]^{-1} .
$$

\section{Total horizontal and vertical deformation caused by combined loads $F_{\|}$and $F_{\perp}$}

When both horizontal and longitudinal forces exist at the same time, there is a combination relation according to the principle of linear superposition, from Eqs.29 and 39 , we have $\Delta_{x \|}+\Delta_{x \perp}=\Delta_{x}$, or $\Delta_{x \|} / R_{s}+\Delta_{x \perp} / R_{s}=$ $\Delta_{x} / R_{s}$, namely

$$
\frac{\Delta_{x}}{R_{s}}=\frac{F_{\|} R_{s}^{2}}{B K_{x \|}\left(\theta_{0}\right)}+\frac{F_{\perp} R_{s}^{2}}{B K_{x \perp}\left(\theta_{0}\right)},
$$

Substituting Eqs 4 and5 into Eq.41, we have the total horizontal deformation and force relation as follows

$$
\frac{\Delta_{x}}{R_{s}}=\frac{1}{2} \frac{F R_{s}^{2}}{B}\left[\frac{1}{K_{x \|}\left(\theta_{0}\right)} \frac{\tan \varphi-\mu}{1+\mu \tan \varphi}+\frac{1}{K_{x \perp}\left(\theta_{0}\right)}\right],
$$

For total vertical deformation $\Delta_{y}$, we have

$$
\frac{\Delta_{y}}{R_{s}}=\frac{F_{\|} R_{s}^{2}}{B K_{y \|}\left(\theta_{0}\right)}+\frac{F_{\perp} R_{s}^{2}}{B K_{y \perp}\left(\theta_{0}\right)},
$$

Substituting Eq.4 and5 into Eq.43, we have the total horizontal deformation and force relation as follows

$$
\frac{\Delta_{y}}{R_{s}}=\frac{1}{2} \frac{F R_{s}^{2}}{B}\left[\frac{1}{K_{y \|}\left(\theta_{0}\right)} \frac{\tan \varphi-\mu}{1+\mu \tan \varphi}+\frac{1}{K_{y \perp}\left(\theta_{0}\right)}\right],
$$

\section{Assembling and disassembling force}

Consider the radius mismatch between buckle and rigid column, that is $R_{c}>R_{s}$, From the deformation figure $\Delta_{x}=R_{c} \sin \varphi-R_{s} \sin \Phi=R_{s}(\alpha \sin \varphi-\sin \Phi)$, so $\frac{\Delta_{x}}{R_{s}}=\alpha \sin \varphi-\sin \Phi$, substitute into the expression (42) to obtain the force $F$

$$
\frac{F R_{s}^{2}}{B}=2 K_{x \|}\left(\theta_{0}\right) \frac{\alpha \sin \varphi-\sin \Phi}{\frac{\tan \varphi-\mu}{1+\mu \tan \varphi}+\frac{K_{x \|}\left(\theta_{0}\right)}{K_{x \perp}\left(\theta_{0}\right)}} .
$$

If ignoring the friction, namely $\mu=0$, the Eq.45 is reduced to

$$
\frac{F R_{s}^{2}}{B}=2 K_{x \|}\left(\theta_{0}\right) \frac{\alpha \sin \varphi-\sin \Phi}{\tan \varphi+\frac{K_{x \|}}{K_{x \perp}\left(\theta_{0}\right)}} .
$$

and for this expression we're going to take the maximum for $\varphi$, if we ignore $K_{x \perp}\left(\theta_{0}\right)$. From the extremum condition $\frac{d F}{d \varphi}=0$, we have

$$
\sin \varphi^{*}=\left(\frac{\sin \Phi}{\alpha}\right)^{1 / 3}
$$

It is worth to mention that [4] has a typo on the above relation as $\varphi^{*}=\left(\frac{\sin \Phi}{\alpha}\right)^{1 / 3}$. We thus can get the maximum assembling force $\stackrel{\alpha}{F}_{A}$

$$
\frac{F_{A} R_{s}^{2}}{B}=2 \alpha K_{x \|} \frac{\left[1-\left(\frac{\sin \Phi}{\alpha}\right)^{2 / 3}\right]^{3 / 2}}{1+\frac{K_{x \|}\left(\theta_{0}\right)}{K_{x \perp}\left(\theta_{0}\right)}\left[\left(\frac{\alpha}{\sin \Phi}\right)^{2 / 3}-1\right]^{1 / 2}}
$$

As you can see, this expression is a modification of the corresponding expressions for Yoshida and Wada [4].

For the disassembly process, it can be considered completely stuck. At this time, the snap fit angle $\varphi \approx \Phi / \alpha$, the disassembly force $F_{D}$ without friction is

$$
\frac{F_{D} R_{s}^{2}}{B}=2 \alpha K_{x \|}\left(\theta_{0}\right) \frac{\sin (\Phi / \alpha)-\alpha^{-1} \sin \Phi}{\tan (\Phi / \alpha)+K_{x \|}\left(\theta_{0}\right) / K_{x \perp}\left(\theta_{0}\right)} .
$$

which is also a modification of the corresponding expressions for Yoshida and Wada [4].

Using relation $\tan \varphi^{*}=\frac{\left(\frac{\sin \Phi}{\alpha}\right)^{1 / 3}}{\sqrt{1-\left(\frac{\sin \Phi}{\alpha}\right)^{2 / 3}}}$, we can also derive the assembling force considering friction

$$
\frac{F_{A} R_{s}^{2}}{B} \approx 2 \alpha K_{x \|}\left(\theta_{0}\right) \frac{\left(\frac{\sin \Phi}{\alpha}\right)^{1 / 3}-\frac{\sin \Phi}{\alpha}}{\frac{\tan \varphi^{*}-\mu}{1+\mu \tan \varphi^{*}}+\frac{K_{x \|}\left(\theta_{0}\right)}{K_{x \perp}\left(\theta_{0}\right)}} .
$$

equivalently

$$
\frac{F_{A} R_{s}^{2}}{B} \approx 2 \alpha K\left(\theta_{0}\right) S(\alpha, \Phi, \mu)\left[1-\left(\frac{\sin \Phi}{\alpha}\right)^{2 / 3}\right]^{3 / 2}
$$


and disassembling force

$$
\frac{F_{D} R_{s}^{2}}{B}=2 \alpha K\left(\theta_{0}\right) \frac{\sin (\Phi / \alpha)-\alpha^{-1} \sin \Phi}{K\left(\theta_{0}\right) / K_{\perp}\left(\theta_{0}\right)-g(\Phi / \alpha,-\mu)}
$$

where

$$
\begin{aligned}
g(\varphi, \mu) & =\frac{\mu-\tan \varphi}{1+\mu \tan \varphi} \\
S(\alpha, \Phi, \mu) & =\frac{\tan \left[\left(\alpha^{-1} \sin \Phi\right)^{1 / 3}\right]}{\frac{K\left(\theta_{0}\right)}{K_{\perp}\left(\theta_{0}\right)}-g\left(\left(\frac{\sin \Phi}{\alpha}\right)^{1 / 3}, \mu\right)} .
\end{aligned}
$$

E. Vertical displacement $u$ driven by vertical force F

From Fig.9, we can see that the vertical displacement $u$ is expressed by

$$
u=R_{s}(1-\cos \Phi)-R_{c}(1-\cos \varphi)-\Delta_{y} .
$$

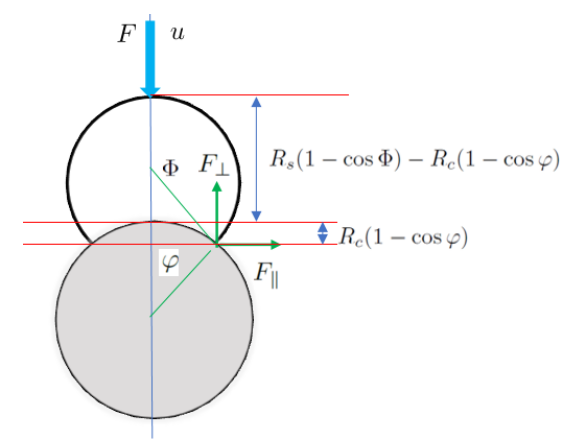

FIG. 9: Vertical displacement $u$

The geometric relation $R_{s} \sin \Phi=R_{c} \sin \varphi$, leads $\operatorname{tocos} \varphi=\sqrt{1-\sin \varphi}=\sqrt{1-\left(\frac{\sin \Phi}{\alpha}\right)^{2}}$, then we have

$$
\begin{aligned}
\frac{u(\Phi)}{R_{s}} & =1-\alpha-\cos \Phi+\sqrt{\alpha^{2}-\sin ^{2} \Phi} \\
& -\frac{1}{2} \frac{F R_{s}^{2}}{B}\left[\frac{1}{K_{y \|}\left(\theta_{0}\right)} \frac{\tan \varphi-\mu}{1+\mu \tan \varphi}+\frac{1}{K_{y \perp}\left(\theta_{0}\right)}\right]
\end{aligned}
$$

\section{IMPROVED THEORY OF MECHANICS OF SNAP FIT ASSEMBLY AND DISASSEMBLY}

Although we have reformulated and improved all expressions for deformation, assembling force and disassembling force of Yoshida and Wada [4]. Unfortunately, because all expressions are implicit forms expressed by $\theta_{0}$, they cannot be written explicitly in terms of initial opening angle $\Phi$. So they are not convenient for application and need further improvement.

From the above analysis, it can be seen that an approximate explicit expression $\theta_{0}=\theta_{0}(\Phi)$ must be found in order to obtain mechanical properties of snap fit. In the previous section, we had the exact solution (13), an explicit expression (6) for Yoshida and Wada [4], and our implicit form (16). They all have their own advantages, and now our question is, how can we use them to get an approximate explicit expression $\theta_{0}=\theta_{0}(\Phi)$ ?

The basic idea is this: since Yoshida and Wada [4] has already proposed an approximate explicit expression (6), we will use our exact solution to modify their expression (6) by the exact solution (13). Then the modified approximate explicit expression is used to derive the other expressions of snap fit assembly and disassembly mechanics.

After comparison with the exact solution, the improved expression is

$$
\theta_{0}=\Phi-\left\{\sin \Phi-\left(1+\frac{3 \epsilon}{2}\right) \Phi \cos [(1-\epsilon) \Phi]+4 \epsilon\right\} \epsilon
$$

In order to compare with the exact solution, we plot expressions (6),(13) and (57) in Fig.10 for different parameters $\epsilon=\frac{F_{\|} R_{s}^{2}}{B}$. It can be seen that the improved expression (57) is better.

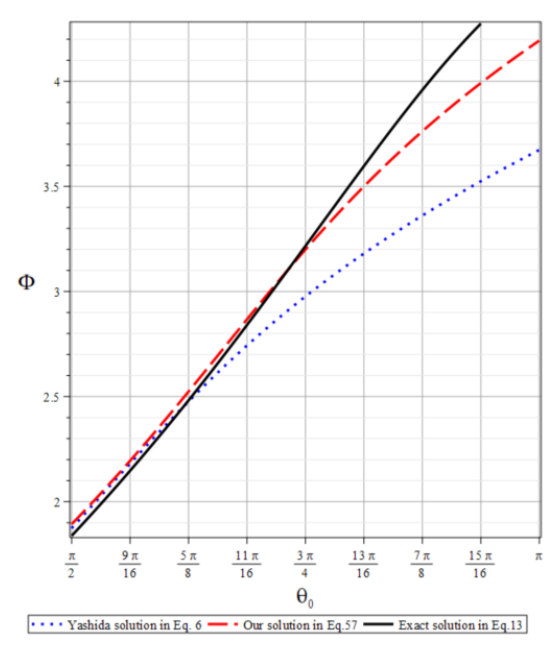

FIG. 10: the comparison of expression(6),(13)and(57), $\epsilon=0.2$

Substitute the improved expression (57) into (23), i.e $K_{x \|}\left(\theta_{0}\right)=\left[\frac{\theta_{0}}{2}-\left(\frac{3}{2} \sin \theta_{0}-\theta_{0} \cos \theta_{0}\right) \cos \theta_{0}\right]^{-1}$, and it approximates to the first order of $\epsilon$

$$
K_{x \|}(\Phi)=\left[\frac{\Phi}{2}-\left(\frac{3}{2} \sin \Phi-\Phi \cos \Phi\right) \cos \Phi\right]^{-1},
$$

Surprisingly, this expression $K_{x \|}(\Phi)$ is exactly the same as the expression Yoshida and Wada [4].

We can modify the corresponding expressions for assembling and disassembling forces in the previous section. Since our approximations are all taken to first-order $\epsilon$, the mounting force deduced by using the expression (57) is

$$
\frac{F_{A} R_{s}^{2}}{B}=2 \alpha K_{x \|}(\Phi)\left[1-\left(\frac{\sin \Phi}{\alpha}\right)^{2 / 3}\right]^{3 / 2}
$$


for the disassembly process, can be considered completely stuck, then the snap fit angle $\varphi \approx \Phi / \alpha$, no friction is the disassembly force $F_{D}$ is

$$
\frac{F_{D} R_{s}^{2}}{B}=2 \alpha K_{x \|}(\Phi) \frac{\sin (\Phi / \alpha)-\alpha^{-1} \sin \Phi}{\tan (\Phi / \alpha)},
$$

also exactly the same as the expressions of Yoshida and Wada [4].

Similarly, we can derive the assembling force taking into account friction

$$
\frac{F_{A} R_{s}^{2}}{B} \approx 2 \alpha K_{x \|}(\Phi) S(\alpha, \Phi, \mu)\left[1-\left(\frac{\sin \Phi}{\alpha}\right)^{2 / 3}\right]^{3 / 2}
$$

and disassembling force

$$
\frac{F_{D} R_{s}^{2}}{B}=2 \alpha K_{x \|}(\Phi) \frac{\sin (\Phi / \alpha)-\alpha^{-1} \sin \Phi}{K_{x \|}(\Phi) / K_{x \perp} \perp(\Phi)-g(\Phi / \alpha,-\mu)}
$$

where

$$
\begin{aligned}
K_{x \perp}(\Phi) & =\left[\frac{1}{2}+\left(1-\Phi \sin \Phi-\frac{3 \cos \Phi}{2}\right) \cos \Phi\right]^{-1} \\
S(\alpha, \Phi, \mu) & =\frac{\tan \left[\left(\alpha^{-1} \sin \Phi\right)^{1 / 3}\right]}{\frac{K_{x \|}(\Phi)}{K_{x \perp}(\Phi)}-g\left(\left(\frac{\sin \Phi}{\alpha}\right)^{1 / 3}, \mu\right)}
\end{aligned}
$$

the assembling and disassembly forces which consider friction are also exactly the same as the expressions for Yoshida and Wada [4].

The vertical displacement $u$ is simplified to the following

$$
\frac{u(\Phi)}{R_{s}}=1-\alpha-\cos \Phi+\sqrt{\alpha^{2}-\sin ^{2} \Phi}-\frac{1}{2} \frac{F R_{s}^{2}}{B} J_{u}(\Phi),
$$

where $J_{u}(\Phi)=\left[\frac{1}{K_{y \|}(\Phi)} \frac{\tan \varphi^{*}-\mu}{1+\mu \tan \varphi^{*}}+\frac{1}{K_{y \perp}(\Phi)}\right]$. [4] derived a similar expression, ie., $\frac{u(\varphi)}{R_{s}} \approx \sqrt{\alpha^{2}-\sin ^{2} \Phi}-\alpha \cos \varphi-$ $\frac{1}{2} \frac{F R_{s}^{2}}{B} J_{u}(\Phi)$, which might have typos.

Although the first order result of our improved expression is exactly the same as that of Yoshida and Wada [4], the affirmation is not wasted but actually makes scientific sense. From the perspective of approximation, it answers the question we raised in the beginning: whether the theoretical result of Yoshida and Wada [4] is credible. So far, we have theoretically verified and confirmed that Yoshida and Wada's theory is credible and correct wihtin the first order approximation of $O(\epsilon)$.

\section{FINITE ELEMENT SIMULATION OF PUSHING ASSEMBLING AND PULLING DISASSEMBLING OF CYLINDRICAL SNAP FIT}

Although we have mathematically verified that the formulations of Yoshida and Wada [4] is credible, we still like to emphasize that the theory is just a linearized approximation theory. In actual situation, the deformation of snap fit we observe is generally nonlinear and large deformation, so we need to conduct further verification from other aspects. Yoshida and Wada [4] did the experiment and put forward a discrete model for numerical calculation, which is consistent with their own theoretical prediction. Yoshida and Wada [4] have not used the finite element method for verification, and we believe that the finite element method is a relatively effective method. In order to make up for the lack of finite element simulation, we conducted a detailed finite element analysis on the assembly and disassembly process of the snap fit.

In order to obtain enough data, we use finite element analysis program ABAQUS to simulate and analyze the

\begin{tabular}{|c|c|c|c|}
\hline radius ratio & length & thickness & opening angle \\
\hline$\alpha$ & $\mathrm{b}(\mathrm{mm})$ & $\mathrm{t}(\mathrm{mm})$ & $\Phi(r a d)$ \\
\hline 1.14 & 20 & 0.3 & 1.9 \\
\hline 1.14 & 20 & 0.3 & 2.0 \\
\hline 1.14 & 20 & 0.3 & 2.1 \\
\hline 1.14 & 20 & 0.3 & 2.2 \\
\hline 1.14 & 20 & 0.3 & 2.3 \\
\hline 1.14 & 20 & 0.3 & 2.4 \\
\hline 1.14 & 20 & 0.3 & 2.5 \\
\hline 1.14 & 20 & 0.3 & 2.6 \\
\hline
\end{tabular}
assembly and disassembly forces of eight cylindrical snap fit models. Snap fit parameters: the radius ratio is $\alpha=$ $R_{c} / R_{s}=1.14$,and semi-cylindrical shells with different opening angles are adopted, as shown in Table I. The finite element model is shown in Fig.11.

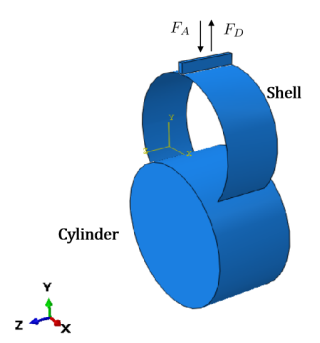

FIG. 11: Finite element model

We established the model according to the radius ratio $\alpha$,opening angle $\Phi$ and material parameters of the buckle in Yoshida and Wada [4]. The simulation process is divided into two steps of assembly and disassembly.During the assembly process, the shell moves down at a speed of $5 \mathrm{~mm} / \mathrm{s}$ until the top of the shell touches the cylindrical surface. After the spacing of $1 s$, the housing will move up at the same speed as $5 \mathrm{~mm} / \mathrm{s}$ (the disassembly process).

(1) Frictionless assembling force: without considering friction, the snap fit with small opening angle $\Phi$ is easier to slide along the surface of the rigid cylinder. At this time, the deformation of small assembling force belongs to small deformation, so the theoretical prediction is relatively close to the finite element results, see Fig. 12. 


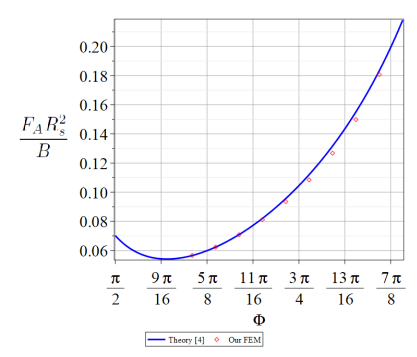

FIG. 12: Type I snap fit ( $\alpha=1.14)$ comparison of assembling forces without considering friction $(\mu=0)$ : Our finite element results are in agreement with Yoshida and Wada [4] expressions $\frac{F_{A} R_{s}^{2}}{B}=2 \alpha K(\Phi)\left[1-\left(\frac{\sin \Phi}{\alpha}\right)^{2 / 3}\right]^{3 / 2}$. Make every effort to guide the assembly and set it to zero,we can get the minimum assembling force of snap fit opening angle $\Phi=1.79 \mathrm{rad} \approx 102^{0}$.

(2) Assembling force with friction: considering friction, the snap fit with $\Phi$ small opening angle is easier to slide along the surface of the rigid cylinder. However, due to friction, the friction resistance increases and an additional force is generated to act on the snap fit. Since this force is opposite to the sliding direction, the deformation of the snap fit becomes larger, and the deformation is no longer a small one. Therefore, for the prediction of assembling force, the finite element result is better than that of the linear theory, see Fig.13.

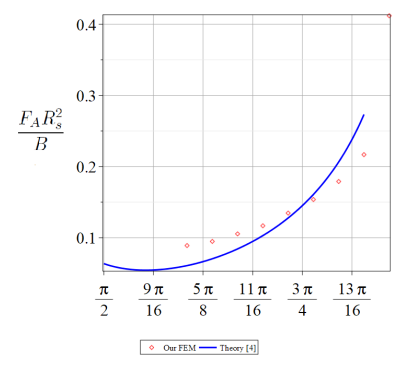

FIG. 13: Type I snap fit ( $\alpha=1.14)$ comparison of assembling forces considering friction $(\mu=0.21)$ : Our finite element results are in agreement with Yoshida and Wada [4] expressions $\frac{F_{A} R_{s}^{2}}{B}=2 \alpha K(\Phi) S(\alpha, \Phi, \mu)\left[1-\left(\frac{\sin \Phi}{\alpha}\right)^{2 / 3}\right]^{3 / 2}$. Make every effort to guide the assembly and set it to zero, we can get the minimum assembling force of snap fit opening angle $\Phi=1.74 \mathrm{rad} \approx 100^{\circ}$.

(3) Frictionless disassembling force: without considering the friction, the linear theoretical prediction is closer to the finite element prediction,see Fig.14.

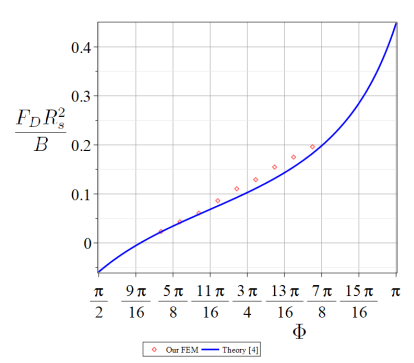

FIG. 14: Type I snap fit $(\alpha=1.14)$ comparison of disassembling forces without considering friction $(\mu=0)$ : Our finite element results are in agreement with Yoshida and Wada [4] expressions $\frac{F_{D} R_{s}^{2}}{B}=2 \alpha K(\Phi) \frac{\sin (\Phi / \alpha)-\alpha^{-1} \sin \Phi}{K\left(\theta_{0}\right) / K_{\perp}\left(\theta_{0}\right)-g(\Phi / \alpha,-\mu)}$

(4) Assembling force with friction: considering friction,the snap fit with $\Phi$ small opening angle is easier to slide along the surface of the rigid column, the deformation of the snap fit is small and the linear theoretical prediction is close to the finite element results; however, for the snap fit with a larger opening angle $\Phi$, it is not easy to slide along the surface of the rigid column. There will be a relatively large deformation before sliding, and the small deformation theory cannot predict it, so the theoretical prediction of disassembling force is less than that of finite element method. In this case, the theoretical prediction without finite element method is good, see Fig.15.

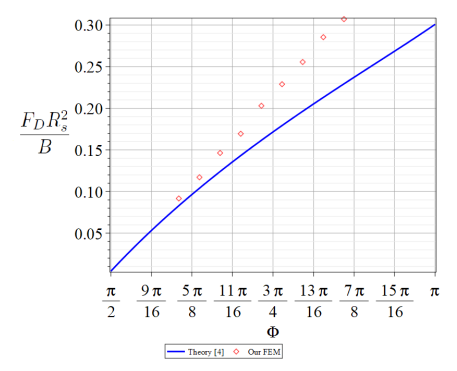

FIG. 15: Type I snap fit ( $\alpha=1.14)$ comparison of assembling forces considering friction $(\mu=0.21)$ : Our finite element results are in agreement with Yoshida and Wada [4] expressions $\frac{F_{D} R_{s}^{2}}{B}=2 \alpha K(\Phi) \frac{\sin (\Phi / \alpha)-\alpha^{-1} \sin \Phi}{K\left(\theta_{0}\right) / K_{\perp}\left(\theta_{0}\right)-g(\Phi / \alpha,-\mu)}$

Our finite element analysis (Figs.12,13,14,15) show that the assembling forces derived from Yoshida and Wada [4] are acceptable. When the initial opening angle is small, the theory is consistent with the finite element method. However, when the initial opening angle is large, the error becomes large. The reason is that the theoretical hypothesis is that the deformation of the snap fit is symmetric and small, but when the initial opening angle is relatively large, the deformation before the snap fit is relatively large and asymmetric. In fact, the linear theory can not be used to simulate those large deformation , so in those cases it is understandable that the FEM is inconsistent with the linear theory.

The finite element simulation results were compared 
with the discrete simulation curves of Type II snap fit in Fig.2(b) and Type II snap fit in Fig.2(d) in Yoshida and Wada [4], as shown in Fig .17. The measured force $F$ is expressed in units $R_{s}^{2} / B$.
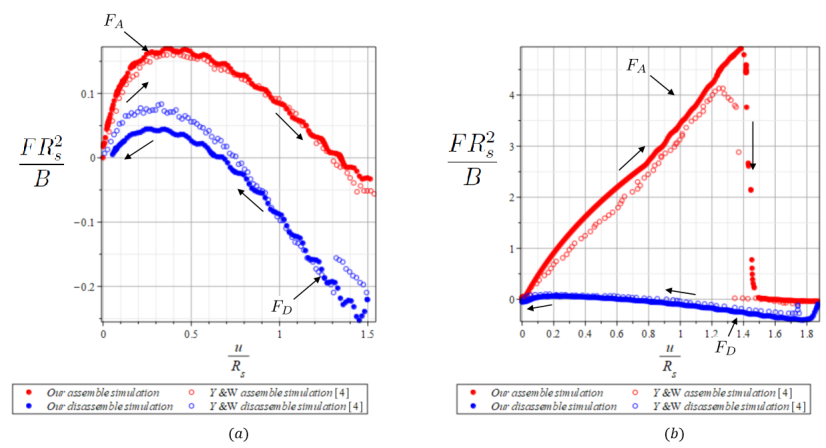

FIG. 16: Finite element simulation comparison diagram of snap fit assembly and disassembly.(a)Type I snap fit,(b)Type II snap fit.

The finite element parameter simulation made by us is basically consistent with the discrete simulation of Yoshida and Wada [4], and the rule of curve variation is completely consistent. As can be seen from the above finite element simulation results, the mechanical asymmetry of the snap fit is mainly closely related to shell geometry $\Phi$.

\section{BENDING DISASSEMBLING ANALYSIS OF CYLINDRICAL SNAP FIT}

In addition to the above pushing assembling and pulling disassembling, we propose a new kind of operation, namely pushing assembling and bending disassembling, which is to use bending snap fit to achieve the purpose of disassembly. Specific bending disassembly is shown in Fig. 17.
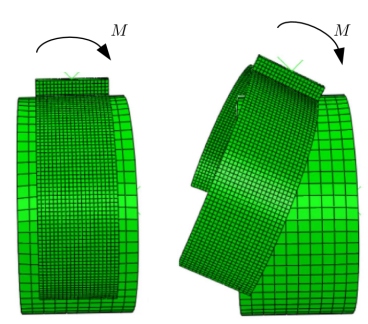

FIG. 17: Bending disassembling

We have not seen any scientific studies on the use of bending to remove the snap fit, and here is the first time we have raised this question. In order to illustrate the problem, we use the 12 finite element models in Table II to study the bending disassembling of cylindrical snap fit. The assembly process is the same as before. In the disassembly process, the shell rotates outward at the Angle of $1 \mathrm{rad}$. The specific finite element model and bending condition are shown in Fig. 17.

After finite element calculation, the specific simulated disassembly bending moment is shown in Table II.The measured moment $M$ is expressed in units of $R_{s} / B$.

TABLE II: Disassembly bending moment of cylindrical snap fit

\begin{tabular}{ccccc}
\hline \multicolumn{6}{c}{ Radius ratio Length } & Thickness & Opening angle Bending moment \\
\hline$\alpha$ & $\mathrm{b}(\mathrm{mm})$ & $\mathrm{t}(\mathrm{mm})$ & $\Phi(\mathrm{rad})$ & $M(N \cdot \mathrm{m})$ \\
\hline 1.14 & 20 & 0.3 & 1.81 & 0.68940 \\
\hline 1.14 & 20 & 0.3 & 1.93 & 1.10210 \\
\hline 1.14 & 20 & 0.3 & 2.00 & 1.64696 \\
\hline 1.14 & 20 & 0.3 & 2.10 & 2.76044 \\
\hline 1.14 & 20 & 0.3 & 2.20 & 2.85505 \\
\hline 1.14 & 20 & 0.3 & 2.30 & 2.62103 \\
\hline 1.14 & 20 & 0.3 & 2.40 & 3.08299 \\
\hline 1.14 & 20 & 0.3 & 2.50 & 3.22582 \\
\hline 1.14 & 20 & 0.3 & 2.57 & 3.65700 \\
\hline 1.14 & 20 & 0.3 & 2.62 & 3.82076 \\
\hline 1.14 & 20 & 0.3 & 2.66 & 3.90040 \\
\hline 1.14 & 20 & 0.3 & 2.75 & 24.40410 \\
\hline
\end{tabular}

In Fig.18, we draw the relationship between the disassembly moment and the opening angle $\Phi$ during bending unloading of the cylindrical snap fit. It is obvious that there is a significant difference between the disassembly bending moment of Type I snap fit and Type II snap fit, which is about 5-40 times. With the increase of the opening angle of the cylindrical snap fit, the required bending moment increases. When the snap fit changes from Type I to Type II, the bending moment increases rapidly. The simulated phenomenon completely conforms to the physical law.

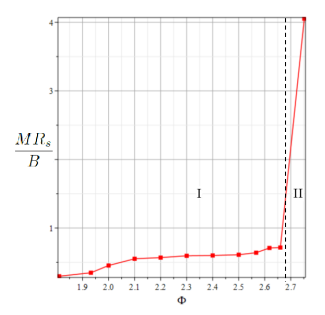

FIG. 18: The relation between disassembly bending moment and opening angle $\Phi$

Therefore, we study the relationship between the disassembly bending moment and the opening angle $\Phi$ during the disassembling of Type I snap fit, and use the scatter diagram of finite element results to fit, as shown in Fig.19. 


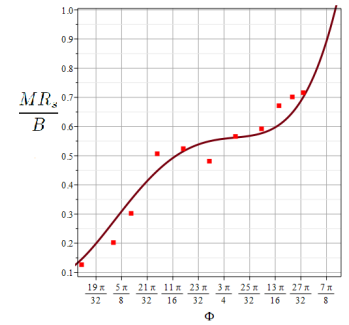

FIG. 19: $\mu=0.21$, disassembly bending moment of Type I snap fit

By data fitting, we get a scaling law of the relation between the disassembly bending moment and the opening angle $\Phi$ :

$\frac{M R_{s}}{B}=5.41 \Phi^{4}-46.89 \Phi^{3}+150.68 \Phi^{2}-212.07 \Phi+110.33$

From our finite element simulation results, it can be seen that the disassembly bending moment of cylindrical snap fit during disassembling is in a power relationship with the opening angle.The smaller the opening angle $\Phi$ is, the smaller the disassembling bending moment is. This conclusion is aimed at the bending disassembling of the snap fit, but its law still conforms to the general physical law of the snap fit.

\section{ASSEMBLY AND DISASSEMBLY EXPERIMENTS OF SNAP FIT}

In order to further prove the correctness of the finite element model of the snap fit established by us, numerical comparison between simulation and experiment is carried out.

The experimental model was obtained by 3D printer, and the model parameters were as follows: $R_{s}=$ $26.3 \mathrm{~mm}, R_{c}=30 \mathrm{~mm}, \alpha=R_{c} / R_{s}=1.14, t=1.1 \mathrm{~mm}$, $b=20 \mathrm{~mm}, \Phi=2.2 \mathrm{rad}$. After the model is made, the electronic universal testing machine (E43.1044) is controlled by microcomputer to conduct the assembly experiment of Type I snap fit, as shown in Fig.20 (see supplementary materials for experimental and simulation videos).

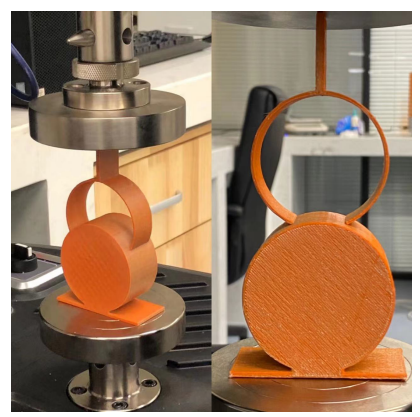

FIG. 20: The experimental setup
We established a finite element model according to the above model parameters, and drew a comparison figure between the simulation data and experimental data of the model, as shown in Fig.21. According to Fig.21, we can intuitively see that the two sets of data are basically the same, and the error does not exclude factors such as friction (friction coefficient is taken from friction experiment). This further proves the correctness of our finite element simulation.

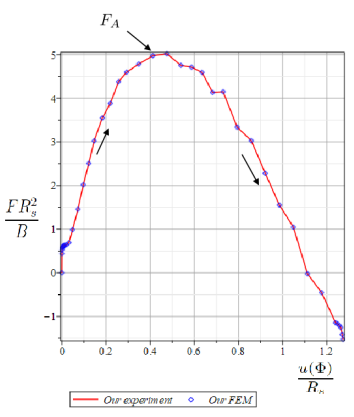

FIG. 21: Simulation data were compared with experimental data

Under the condition that the finite element model is correct, we extract the assembling force and disassembling force of 8 models with different opening angle $\Phi$ in Table I,and redraw the data of $\mu=0.21$ in the form of $\Phi v s\left|F_{D}\right| / F_{A}$, which is defined as "locking rate" [7] in Ref [4].Verify the relationship between the mechanical asymmetry of the snap fit and the opening angle $\Phi$, as shown in Figure.56.

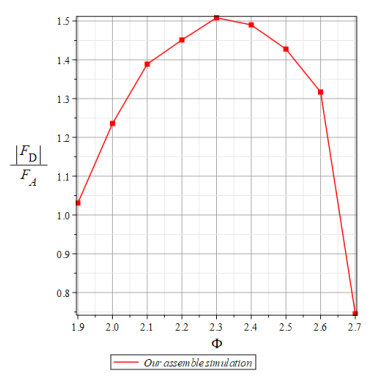

FIG. 22: Simulation data were compared with experimental data

Draw conclusions in Yoshida and Wada [4]: When the $2 \leq \Phi \leq 2.6$, can achieve the ideal conditions for $\left|F_{D}\right| / F_{A}>1$, this is the Type I snap fit height overlapping. The trend for other typical $\mu$ value effectively, $\left|F_{D}\right|$ and $F_{A}$ relative size can only be adjusted by shell geometry.However, it is obvious from the simulation that when $t=0.3 \mathrm{~mm}$ of shell geometry, both $\Phi=1.9 \mathrm{rad}$ and $\Phi=2.6 \mathrm{rad}$ can reach the ideal condition $\left|F_{D}\right| / F_{A}>1$. This not only shows that the ideal condition overlaps with the Type I snap fit,but also shows that the shell thickness has a certain influence on the relative size of sum. Thus, the relative sizes of $\left|F_{D}\right|$ and $F_{A}$ can be 
adjusted by the thickness of shell geometry $t$ and the opening angle $\Phi$.

It is found that under different friction conditions, the relationship between the disassembly bending momen$\mathrm{t}$ and the opening angle $\Phi$ obtained by finite element simulation is consistent, the only difference is that the coefficient in front is not consistent, which indicates that friction does not affect the physical law between the disassembly bending moment and the opening angle $\Phi$.

In Fig.18, it can be clearly seen that $M$ increases with the increase of $\Phi$ and diverges with $\Phi \rightarrow \Phi_{\mid-\|}$, at which point the system changes from Type I to Type II. Therefore, we can adjust the size of buckle opening angle $\Phi$, so as to achieve the purpose of snap fit design.

\section{CONCLUSIONS}

In light of Yoshida and Wada [4], the assembly and disassembly mechanics of the snap fit is carefully reformulated, finite element analysis and experiment have been carried out. Our reformulations confirmed the correctness and credible of their approximations. We have proposed a new way of disassembly, namely disassembly by bending. Studies also reveal that the asymmetry is the key mechanism behind of the easy assembly and difficulty disassembly of snap fit. The symmetry breaks is emerged from the interaction between elasticity, geometry and cylinder friction. Although this paper only focuses on the cylindrical snap fit, the method in this paper is instructive to the research of other snap fit configurations. Because of the wide application of snap fit in daily life, especially some industrial snap fit need to know the exact assembly and disassembly force, from a scientific point of view to study its performance and predict its mechanical properties, for the design and production of buckle is very meaningful. This paper is a part of our research on snap fit mechanics, and the results of other shape snap fit mechanics analysis will be reported in the future.

Acknowledgement: The authors wish to express their appreciation to the financial support from Xian University of Architecture and Technology (Project no: 002/2040221134).

Conflict of interest The author declares that he has no known competing financial interests or personal relationships that could have appeared to influence the work reported in this paper.

Data availability The data that support the findings of this study are available from the corresponding author upon reasonable request.
[1] Bayer Corporation. Snap fit Joints for Plastics: A Design Guide (Polymer Division, Pittsburgh, PA, 1996).

[2] V. T. Moy, E. L. Florin, and H. E. Gaub. Intermolecular Forces and Energies Between Ligands and Receptors. Science 266, 257(1994).

[3] M. Romano, D. A. Friedman, and T. J. Shay. Laboratory Experimentation of Autonomous Spacecraft Approach and Docking to a Collaborative Target. J. Spacecr.Rockets 44, 164 (2007).

[4] K. Yoshida and H. Wada. Mechanics of a snap fit. Phys.Rev.Lett. 125, 194301 (2020)

[5] Supplemental materials at http://link.aps.org/ supplemental/10.1103/PhysRevLett.125.194301

[6] Abramowitz, M., and Stegun, I., eds. Handbook of Mathematical Functions. New York: Dover publications (1972).

[7] G. Suri and A. F. Luscher. Structural Abstraction in Snap-fit Analysis. J. Mech. Des. 122, 395 (2000).

[8] R. C. Benson. The Deformation of a Thin Incomplete, Elastic Ring in a Frictional Channel . J. Appl. Mech. 48, 895 (1981).

[9] R. C. Benson. Stick/Slip Conditions for a Thin, Incomplete, Elastic Ring Impinging on a Frictional Barrier. J. Appl. Mech. 49, 231 (1982).

[10] C.-W. Liu and J.-S. Chen. Effect of friction on the planar elastica constrained inside a circular channel with clearance. Int. J. Solids Struct. 50, 270(2013).

[11] B. Roman and A. Pocheau. Postbuckling of bilaterally constrained rectangular thin plates. J. Mech. Phys. Solids $50,2379(2002)$.
[12] Q. S. Nguyen. Instability and friction Instabilité et frottement. C. R. Mec. 331, 99 (2003).

[13] A. Rafsanjani, A. Akbarzadeh, and D. Pasini. Multistable Architected Materials for Trapping Elastic Strain Energy. Adv. Mater.27, 5931 (2015).

[14] E. Popova and V. Popov. The research works of Coulomb and Amontons and generalized laws of friction. Friction $3,183(2015)$.

[15] J. Ji, K.-M. Lee, and S. Zhang. The research works of Coulomb and Amontons and generalized laws of friction. J. Mech. Des. 133, 121004 (2011).

[16] L. Wu, X. Xi, B. Li, and J. Zhou. Multi-Stable Mechanical Structural Materials. Adv. Eng. Mater. 20, 1700599 (2018).

[17] B. R. Mose, I.-S. Son, J.-W. Bae, H.-G. Ann, C. Y. Lee, and D.-K. Shin. Modified analytical method to calculate the assembly and separation forces of cantilever hooktype snap-fit. J. Mech. Eng. Sci. 233, 5074 (2019).

[18] D. Matsumoto, T. G. Sano, and H. Wada. Pinching an open cylindrical shell: Extended deformation and its persistence. Europhys. Lett. 123, 14001 (2018).

[19] B. Audoly and Y. Pomeau, Elasticity and Geometry (Oxford University Press, New York, 2010).

[20] D. P. Holmes. Elasticity and stability of shape-shifting structures. Curr. Opin. Colloid Interface Sci. 40, 118 (2019).

[21] P. R. Bonenberger, The First Snap-Fit Handbook(3rd ed.), Hanser Publishers, Munich (2016) 